\title{
Lateral Superlattices: Magnetoresistance, Hall Effect and Commensurate Orbits
}

\section{Dieter Weiss}

Bellcore, Red Bank, New Jersey 07701, USA \& Max-Planck-Institut für Festkörperforschung, D-7000 Stuttgart 80, Germany

Summary: The electron motion in a two-dimensional periodic potential and a perpendicular magnetic field, leads to interesting commensurability phenomena. Using nowadays micropatterning techniques, both weak $\left(V_{0}<<E_{F}\right)$ and strong $\left(V_{0}>E_{F}\right)$ periodic potentials can be superimposed upon a two-dimensional clectron gas. In the weak-modulation limit magnetoresistance oscillations periodic in $1 / B$ reflect the commensurability between cyclotron orbit diameter $2 R_{c}$ and the superlattice period. A strong modulation acts predominantly as an array of periodic scatterers. The repulsive scattering potentials seem to trap electrons on commensurate orbits: the reduced carrier density manifests itself in peaks in the magnetoresistance, aperiodic in $1 / B$, and non-quantized plateaus in the Hall resistance.

\section{Introduction}

An artificial periodic potential superimposed upon a two-dimensional clectron gas (2DEG) leads to interesting magnetotransport effects due to the interplay of two length scales, the cyclotron radius $R_{c}$ at the Fermi energy $E_{F}$ and the artificial period $a$. In the case of a two-dimensional periodic potential the flux through the unit cell is expected to play a crucial role: the number of allowed energy levels for Bloch electrons depends on the flux $B a^{2}$ penetrating one unit cell. [1]. In real crystals the observation of such effects is out of the range of possibility since the magnetic field providing one flux quantum, $h / e$, is of order $10^{5} \mathrm{~T}$ for $a \sim 0.2 \mathrm{~nm}$. Artificially manufactured periodic potentials can overcome this problem and make this quantum mechanical regime accessible to experiment. Todays lithographic techniques allow the fabrication of lateral superlattices with periods $a$ as small as $100 \mathrm{~nm}$ : hence the magnetic ficld required for one flux quantum to penetrate a unit cell is reduced by several orders of magnitude ( $\sim 0.4 \mathrm{~T}$ for $a=100 \mathrm{~nm})$. A few concepts to describe magnetotransport in a homogeneous two-dimensional gas are sketched below. This theoretical "tools" are used later on to model magnetotransport coefficients in the presence of artificially periodic potentials. At low magnetic fields, when Landau quantization plays no 
role, the magnetoresistance of a two-dimensional clectron gas can be characterized within the Drude model [2]. The simple formulas within this model are usually used to determine the density $n_{s}$ and mobility $\mu=e \tau / m^{*}(e=$ elementary charge, $m^{*}=$ cffective clectron mass) of the conduction clectrons. Without scattering the electrons perform circular orbits with cyclotron frequency $\omega_{c}=e B / m^{*}$. Scattering processes are described by the relaxation time $\tau$ and for $\omega_{c} \tau<2 \pi$ scattering terminates the circular motion before a full orbit is completed. For $\omega_{c} \tau>>2 \pi$ and for low enough temperature $T\left(\hbar \omega_{c}>>k T\right)$, the clectron energy spectrum becomes discretc. A free electron gas without impurities occupies the highly degenerate Landau levels (LL's) which are seperated by the cyclotron energy $\hbar \omega_{c}$. The occupancy of each level increases linearly with $B$. Scattering processes lead to a collision broadening of those energy levels. Within the selfconsistent Born approximation (SCBA), the simplest way of taking into account randomly distributed short range scatterers, the LL's are described by semi-ellipses [3] with a linewidth connected to the relaxation time $\tau: \Gamma \propto \sqrt{\omega_{c} / \tau}$. In higher order calculations the semi-elliptical shape is replaced by Gaussians [4]. This discrete energy spectrum is the origin of Shubnikov-de Haas oscillations as well as of the quantum Hall effect [5]. In the following sections, magnetotransport in a 2DEG with superimposed weak and strong periodic potentials is studicd. A new class of oscillatory phenomena emerge, but experiments verifying directly the peculiar "Hofstadter butterfly" still remain to be done.

\section{Weak and Strong Periodic Potentials: Fabrication}

Holographic in situ illumination and clectron beam lithography is used to superimpose a periodic potential upon a high mobility 2DEG in GaAs-AlGaAs heterojunctions. The high mobility ensures that the electron mean free path $\ell=\tau v_{F}$, where $v_{F}$ is the Fermi velocity, is much longer than the artificial periodicity. The clectron mobility of a typical sample was of order $1 \times 10^{6}$ $\mathrm{cm}^{2} / V s$ and corresponds for carricr densitics of order $2 \times 10^{11} \mathrm{~cm}^{-2}$ to an clastic mean free path of $\sim 10 \mu \mathrm{m}$. Holographic illumination creates a soft periodic potential with a potential amplitude $V_{0}$ much smaller than the Fermi energy $E_{F}$ in the 2DEG. The method, schematized in Fig. 1, relies on the persistent photoconductivity effect in selectively doped GaAs-AlGaAs heterojunctions at low temperatures [6]. A spatially modulated photon flux, achieved by the interference of two plane waves on top of the sample surface (Fig. 1(a)), results in a spatial modulation of ionized decp Si-donors (Fig. 1(b)). Exposure times in the millisecond range are suficient to "write" the pattern at liquid helium temperatures. After exposure, the two-dimensional electron gas moves under the influence of a modulated and immobile positive background charge. A 

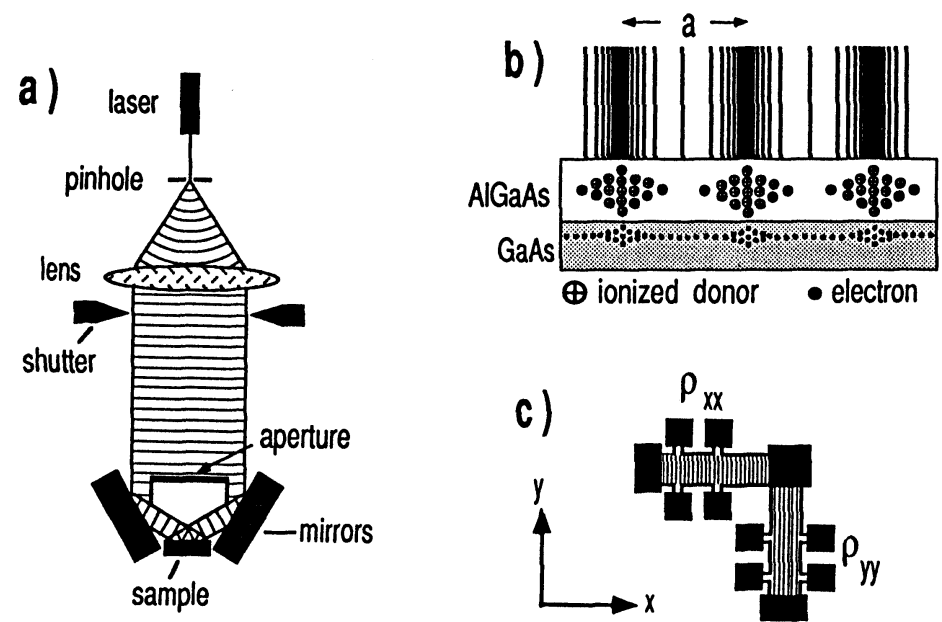

Fig. 1 Two interfering laser beams (a) produce a pattern of dark and bright fringes resulting in a space charge modulation in the underlaying $\mathrm{AlGaAs}$ layer (b). The period $a=\lambda / 2 \sin \Theta$ depends on the wavelength $\lambda$ and the angle of incidence $\Theta$ (with respect to the surface normal). The sample geometry in (c) allows to investigate both, $\rho_{x x}$ and $\rho_{y y}$.

maximum amplitude $V_{0} \sim 0.9 \mathrm{meV}$ for this method has been reported [7]. The induced periodicities are on the order of the wavelength of the interfering beams. The largest period made was $382 \mathrm{~nm}$, the smallest $282 \mathrm{~nm}$. One- and twodimensional (1D- and 2D) lateral periodic potentials can be defined [7]. The pattern is crased when the sample is warmed up to room temperature.

An alternative method to create lateral superlattices is to use electron beam lithography [8]. The fabrication process is sketched in Fig. 2. Holes written in the clectron beam sensitive resist (Fig. 2(a)) define the pattern: a weak modulation is accomplished by covering the patterned region with an evaporated Au-layer which is used as a top gate (Fig. 2(b)). Etching the holes through the 2DEG, on the other hand, results in a strong modulation (Fig. 2(c)). An electron micrograph of such an antidot array is displayed in Fig. 2(d). For the antidot structure it is important to cnsure that the effective hole diameters, $d$, including the depletion regions around the holes do not overlap [8]. The distance between the 2DEG and the sample surface, typically of order $100 \mathrm{~nm}$, determines the shortest period which can be imposed effectively upon the 2DEG. 

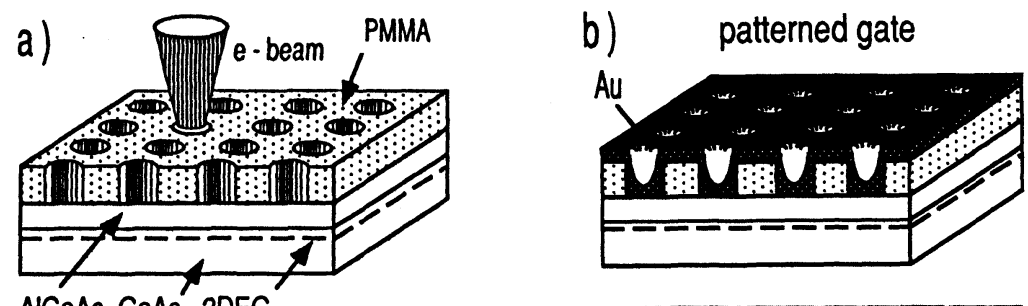

AlGaAs GaAs 2DEG
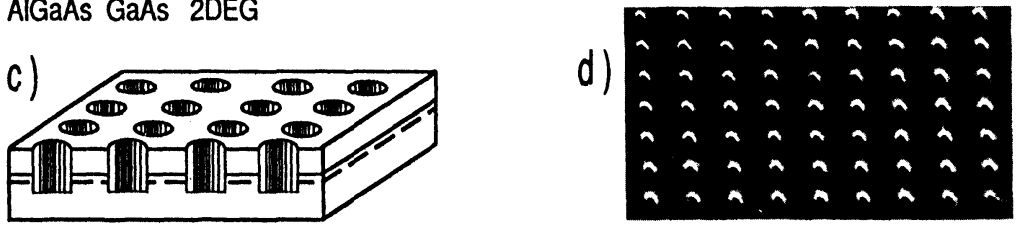

Fig. 2 Sketch of c-bcam patterning (a), and the rcalization of weak (b) and strong (c) periodic potentials. Periods down to $100 \mathrm{~nm}$ have been fabricated. The electron micrograph (d) displays an antidot superlattice with $300 \mathrm{~nm}$ period.

\section{Weak 1D-Modulation}

Magnetotransport experiments in a one-dimensional weak periodic potential highlight the commensurability between $R_{c}$ and the period $a$ and illustratc the two conductivity contributions which govern the weak modulation limit. The first experimental results have been obtained using the in-situ holographic illumination method [6]. Characteristic data are displayed in Fig. 3. The usual Shubnikov-de Haas $(\mathrm{SdH})$ oscillations, periodic in $1 / B$ and reflecting the carricr density $n_{s}$, appear at about $0.5 \mathrm{~T}$. Characteristic for the $1 \mathrm{D}$-modulated systems, however, are the low-ficld oscillations also periodic in $1 / B$ (inset Fig. 3), but with a periodicity which depends also on the period $a$ of the modulation: minima in $\rho_{x x}$ are determined by the commensurability condition

$$
2 R_{c}=(\lambda-1 / 4) a \quad \lambda=1,2,3, \ldots
$$

with the cyclotron radius at the Fermi cnergy, $R_{c}=v_{F} / \omega_{c}=\hbar \sqrt{2 \pi n_{s}} / e B$. The dependence of the oscillation period $\Delta(1 / B)$ on carricr density $n_{s}$ and period $a$ according to Eq. (1) has been verificd in many laboratorics [6, 9...12]. Fig. 3 shows that $\rho_{y y}$ (denoting current flow parallel to the modulation) also displays oscillations: their phase with respect to $\rho_{x x}$, however, is shifted and maxima are observed where $\rho_{x x}$ has minima. The anisotropic oscillatory behaviour reflects different conductivity contributions depending on the direction of current flow with respect to the $1 \mathrm{D}$-modulation. Both effects originate from a modified Landau level energy spectrum: a modulation potential transforms the discrete 


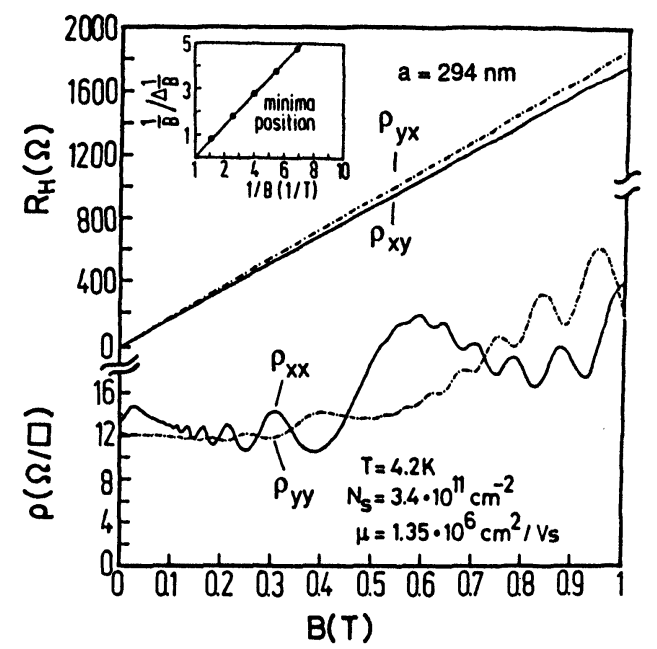

Fig. 3 Magnetoresistance parallel $\left(\rho_{y y}\right)$ and perpendicular $\left(\rho_{x x}\right)$ to a IDmodulation in $\mathrm{x}$-direction. The $1 / B$ periodicity of the low ficld-oscillations is demonstrated in the inset. The typical phaseshift $\pi$ between $\rho_{x x}$ and $\rho_{y y}$ reflects different conductivity contributions (a similar antiphase behavior of the $\mathrm{SdH}$ oscillations emerging above $0.5 \mathrm{~T}$ is accidentally caused by a slightly differing carricr density). No structure in the low-field Hall resistance is resolved (from $[6])$.

LL's into Landau bands. For wcak potentials $\left(V_{0}<<\hbar \omega_{c}\right)$ the band formation can be understood in first order perturbation theory. A cosine potential $V_{0} \cos (K x)$ with period $a=2 \pi / K$ in $x$-direction forms Landau bands given by

$$
E_{n}\left(x_{0}\right)=(n+1 / 2) \hbar \omega_{c}+V_{0} U_{n}(X) \cos \left(K x_{0}\right) .
$$

In contrast to a regular $2 \mathrm{DEG}$, the encrgy eigenvalues now depend on the center coordinate $x_{0}=-l^{2} k_{y}$ with $l=\sqrt{\hbar / e B}$, the magnetic length, and the wavevector $k_{y} . U_{n}(X)$ is an oscillatory function of the $L L$ index $n$ as well as of the argument $X=K^{2} l^{2} / 2$ and determines the bandwidth. The zeros of $U_{n}$ are given by Eq. (1) [13] : for these commensurate magnetic field values the perturbation potential is averaged out and an unperturbed Landau level remains. The potential avcrage is taken over the spatial extent of the wavefunction, approximately given by the classical cyclotron radius $R_{c} \approx l \sqrt{2 n+1}$. It is here were the commensurability between the magnetic length $l$ and the period $a$ enters. The dependence of the Landau level width on the center-coordinate $x_{0}$ (a) and the magnetic field (b) is shown in Fig. 4. Dependent on the quantum number $n$, broad bands alternate with narrow bands and the density of states consequently oscillates over an 

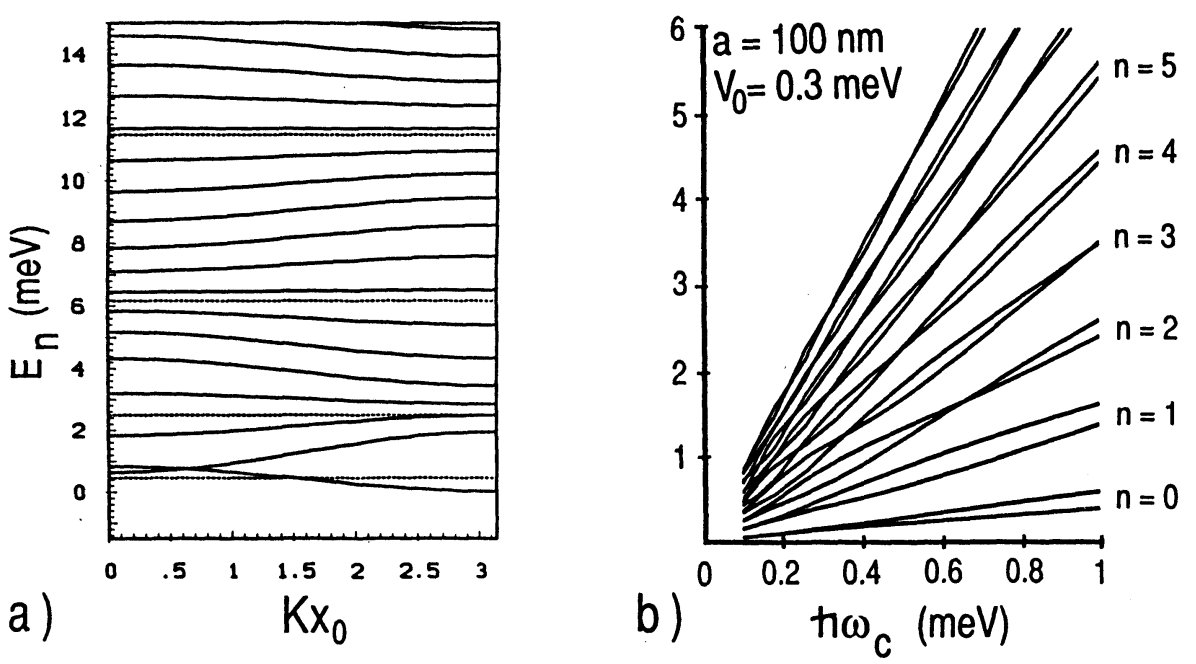

Fig. 4 (a) Landau band dispersion for $B=0.5 \mathrm{~T}, V_{0}=1.5 \mathrm{mcV}$, and $a=100 \mathrm{~nm}$ according to Eq. (2). The flat band situation is marked by the dotted lines (from[13]).

(b) Band width oscillations as function of $B \propto \hbar \omega_{c}$.

energy range large compared to the cyclotron separation $\hbar \omega_{c}$. This is the reason of the weak temperature dependence of the low-ficld oscillations observed in cxperiment $[6,14]$. The oscillating bandwidth within the LL's is displayed in Fig. 4(b); the density of states increases when the linewidth becomes narrower. In Fig. 4 broadening of the LL's due to scattering processes has not been included. The resolution of modulation induced effects requires that the collision broadening is smaller than the modulation broadening. The effect of randomly distributed scatterers on the collision broadening again can be quantitatively described by a linewidth $\Gamma$ within the SCBA formalism $[13,15]$. The magnetocapacitance, measured between a top gate and the 2DEG, is directly related to the thermodynamic density of states [16] and has been used as experimental tool to verify the energy spectrum given by Eq. (2) $[17,18]$. This energy spectrum causes all the oscillatory phenomena in the magnetotransport cocficients. Only a sketch of the transport theory by Zhang and Gerhardts [15], based on Kubo's formulas, can be presented here. Within this approach the conductivity of a periodically modulated 2DEG depends on the square of the density of states at the Fermi energy in just the same analytic manner as for the homogeneous 2DEG [3]:

$$
\sigma_{\mu \mu} \propto \int d E(-\partial f / \partial E)[D(E)]^{2}
$$


where the index $\mu$ stands for the $x$ - or $y$-direction and $\partial f / \partial E$ is the derivative of the Fermi distribution function. In a magnetic ficld the $x x$-component of the conductivity is directly related to the $y y$-component of the resistivity tensor and for $\omega_{c} \tau>>1, \sigma_{x x} \propto \rho_{y y}$ and $\sigma_{y y} \propto \rho_{x x}$ holds (sce, c.g. [15]). For a homogeneous 2DEG, Eq. (3) gives rise to the Shubnikov-de Haas oscillations [3]. For the modulated 2DEG, maxima of the amplitude-modulated DOS lead to peaks in $\sigma_{\mu \mu}$ and consequently the modified density of states in the ID-periodic case modulates the height of the SdH-oscillations. If the thermal smearing $k T$ becomes comparable to $\hbar \kappa_{c}$ the SdH-oscillations are smeared out but the lowficld oscillations remain since the modulation of the DOS maxima takes place on an energy scale large compared to $\hbar \omega_{c}$ (Fig. 4). The conductivity $\sigma_{\mu \mu}$ (Eq. (3)) is isotropic in $x$ - and $y$-direction with maxima for hat Landau bands given by $\mathrm{Eq}$. (1). Eq. (3) describes the maxima position in $\rho_{y y}$ (Fig. 3) correctly, but not the more dominant oncs in $\rho_{x x}$, with their phase shifted by $\pi$. If the modulation is in $x$-direction the $k_{y}$-dispersion of the Landau bands opens up an additional conductivity channcl $[13,9]$ : a non-vanishing group velocity $v_{y} \propto d E_{n} / d k_{y}$ causes enhanced conductivity in $y$ but not in $x$-direction. This gives rise to a band conductivity contribution $\Delta \sigma_{y y} \propto \Delta \rho_{x x}$ which oscillates in phase with $v_{y}$ when the energy bands are swept through the Fermi level with increasing magnetic ficld. Minima in $\Delta \rho_{x x}$ correspond to hat bands since then $d E_{n} / d k_{y}=0$ : this bandconductivity contribution now gives minima in $\rho_{x x}$ while $\rho_{y y}$, not influenced by this mechanism, displays maxima duc to the DOS maxima (denoted in the following as oscillating scattering rate contribution since the conductivity process is due to clastic scattering processes at the Fermi energy and depends therefore on the DOS). The results of calculated traces of $\rho_{x x}$ and $\rho_{y y}$ illustrate their antiphase bchavior (Fig. 5) and summarize the magnetotransport in a weak 1D-periodic potential [15]. A weak oscillatory behavior is also predicted for the Hall resistivity $\rho_{x y}$, but has not been resolved so far experimentally (sec c.g. Fig. 3)

The energy spectrum of the 1D-modulated systems is expected to influence other physical propertics of the two-dimensional electron gas: shifts in the cyclotron resonance frequency [19], oscillatory effects in the thermoclectric transport coeflicients [20], or the magnetoplasmon spectrum [21] have been predicted. Apart from dc-magnetotransport, oscillations in the microwave conductivity have been reported [22].

An illustrative description of the bandconductivity oscillations has been given by Beenakker using a semi-classical approach [23]. He noticed that the square of the drift velocity $\left\langle v_{d}\right\rangle^{2}$ in $y$-direction, caused by the electric fields connected to the periodic potential in $x$-direction $(\boldsymbol{E} \times \boldsymbol{B}$ drift), and averaged over all possible center coordinates is finite and docs only vanish for $2 R_{c}=(\lambda-1 / 4) a$. Within a Boltzmann transport picture the oscillatory behavior of $\rho_{x x}$ (but not $\rho_{y y}$ ) can be described. This picture ignores Landau level quantizations and is applicable only 


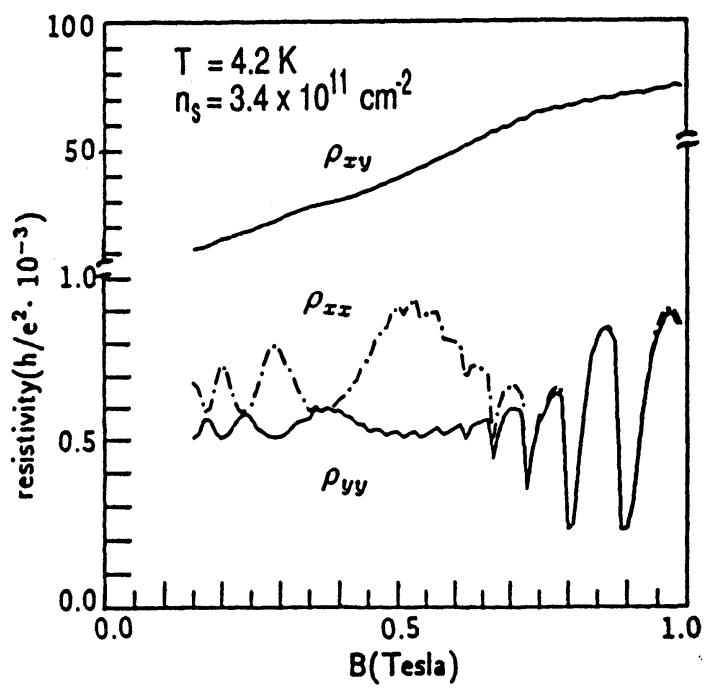

Fig. 5 Calculated resistivity in units of $10^{-3} h / e^{2}=25.8 \Omega$. The parameters in the calculation arc $V_{0}=0.25 \mathrm{mcV}, a=294 \mathrm{~nm}$, and $\Gamma=0.056 \times \sqrt{B[T]} \mathrm{mcV}$. Whilc $\rho_{x x}$ is dominated by the bandconductivity contribution with minima described by Eq. (1), $\rho_{y y}$ follows the density of states oscillations. Around $0.5 \mathrm{~T}$ SdH oscillations commence (from [15]).

if the temperature is high enough $\left(k T \sim \hbar \omega_{c}\right)$. Extending Beenakkers classical calculation to the 2D-periodic case gives exactly the same result: the calculated bandconductivity $\Delta \sigma_{y y}$ is independent of the additional modulation in $y$-direction and again minima in $\rho_{x x}$ are expected when Eq. (1) holds.

\section{Weak 2D-Modulation}

Mcasurements of $\rho_{x x}\left(=\rho_{y y}\right)$ in weakly 2D-modulated 2DEG's, defined by the superposition of two subsequent holographic illuminations, are displayed in Fig. 6. Contrary to the quasi-classical expectation, discussed above, it is obvious that the bandconductivity contribution is suppressed in the presence of a $2 \mathrm{D}$-periodic potential (solid lines): maxima in $\rho_{x x}$ arc observed for flat bands (Eq. (1)), marked by arrows in Fig. $6[7,24]$. High mobility samples are essential to observe this effect; here we used samples with $\mu \geq 1.2 \times 10^{6} \mathrm{~cm}^{2} / V s$, corresponding to a linewidth $\Gamma \leq 0.12 \times \sqrt{B[T]} \mathrm{meV}$ within the SCBA formalism.

The effect of a perturbing 2D-periodic potential $V(x, y)=V_{o}(\cos K x+\cos K y)$ on an initially pure Landau state leads to an intricatc encrgy spectrum [25]. If the period $a$ is the same in both lateral directions the width of the LL's can be 


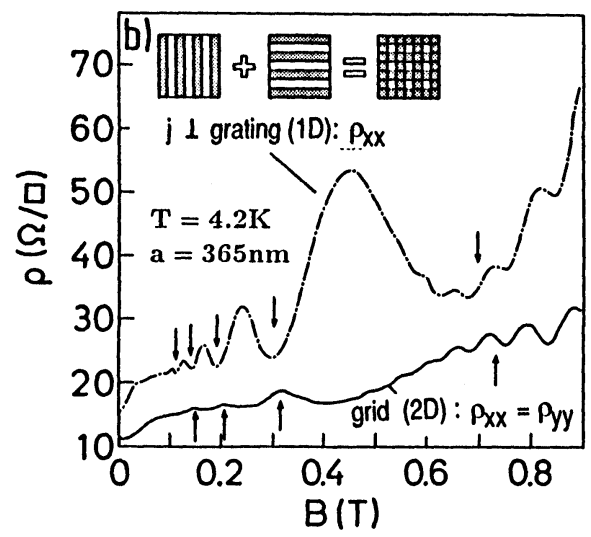

Fig. 6 Magnetoresistance in a 1D- and 2D-periodic potential. Two subsequent holographic in-situ illuminations, with the sample rotated by $90^{\circ}$, creatc the pattern sketched in the inset. The arrows mark the nat band condition (Eq. (1)) and are determined by the carrier density taken from the periodicity of the SdHoscillations. The 2D-periodic potential suppresses the bandconductivity, and the oscillations duc to the oscillating scattering rate (with maxima at the arrow position) dominate (from $[7,24]$ ).

described by the same bandwidth $U_{n}(X)$ as in the $1 \mathrm{D}$-modulated systems (sec, c.g., Fig. 4(b)). The additional modulation in $y$-direction couples Landau states with center coordinates differing by integer multiples of $l^{2} K$. The Landau bands split into $p$ subbands if

$$
B a^{2} / \Phi_{0} \equiv a^{2} / 2 \pi l^{2}=p / q,
$$

i.c., if the flux $B a^{2}$ per unit cell is a rational multiple of the flux quantum $\Phi_{0}=h / e$. Eq. (4) describes an additional commensurability condition. The graphical representation of the energy spectrum is known as Hofstadter's butterny [H]. For real physical systems again random impurity scattering must be taken into account $[24,26]$ : the resulting level broadening destroys the fine structure but the coarse features, connected to small values of $p$, remain for small enough collision broadening. As an example, the calculated density of states [26] is shown as a function of encrgy and magnetic ficld (Fig. 7) assuming a collision broadening $\Gamma=0.035 \times \sqrt{B[T]} \mathrm{mcV}$. Additional gaps, best seen for $n=0$, split each individual LL into subbands; the effect is most pronounced when the modulation broadening, described by $U_{n}(X)$, is at maximum and when only a small number of nux quanta penetrate the unit cell. The Landau level subband splitting reduces drastically the bandconductivity contribution if the collision 


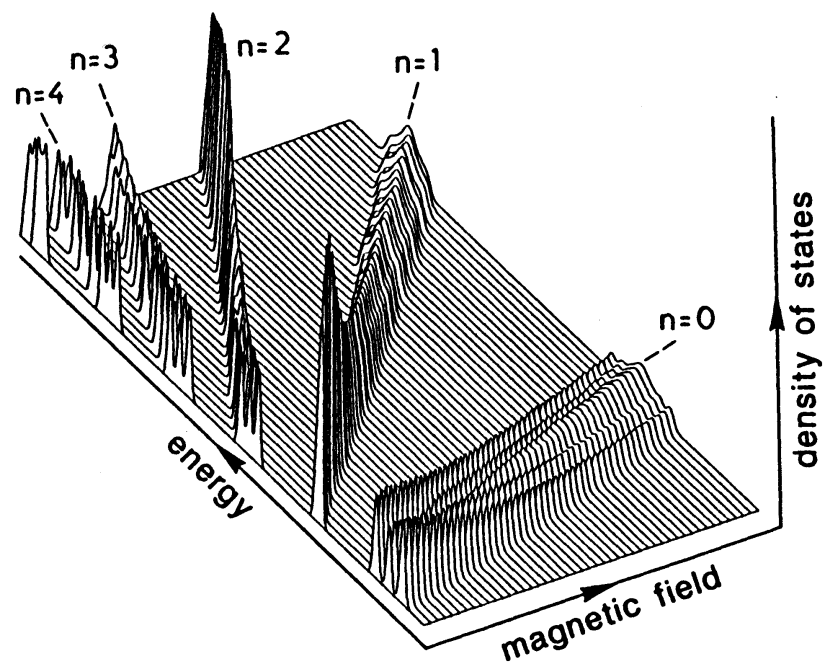

Fig. 7 Density of states for a 2D-modulated 2DEG with $V_{0}=0.25 \mathrm{mcV}$, and $\Gamma=0.035 \times \sqrt{B[T]} \mathrm{meV}$, for $0.5 \mathrm{~T} \leq B \leq 1.65 \mathrm{~T}$ and $0 \leq E \leq 5 \mathrm{mcV}$. The magnetic ficld range corresponds to a change from $\sim 3$ to 9 hux quanta per unit cell (from [26]).

broadening $\Gamma$ is small enough. As a consequence, the oscillating scattering rate can dominate the bandconductivity contribution $[24,26]$. A phase shift of $\pi$ with respect to the oscillations expected quasi-classically results and is observed in Fig. 6. On the other hand, the bandconductivity is expected to dominate for increased $\Gamma$. Experimentally we find the bandconductivity in "low-mobility" samples inded to be the dominant contribution: minima in the envelope of the SdH oscillations are observed for flat bands (Fig. 8(a)). A gate voltage, applied to the microfabricated gate, (sce, c.g., Fig. 2(b)) allows to vary the carricr density, mobility and $V_{0}$. Increasing the mobility with applied positive bias to over $2 \times 10^{6} \mathrm{~cm}^{2} / \mathrm{Vs}$ results again in the suppression of the bandconductivity and reproduces the result of Fig. 6 in a different kind of device and at millikelvin temperatures. The absence of the bandconductivity contribution in high mobility clectron systems, not expected from the semi-classical picture, is interpreted as indirect manifestation of the Hofstadter energy spectrum. The direct manifestation still remains to be shown: the fact that the SdH oscillations in Fig. 8(b) show no internal structure, although the temperature secms low enough $(\sim 5 \mu \mathrm{cV})$ to "scan" the Landau bands, indicates the importance of mesoscopic fluctuations of the electrostatic potential in this system [26]. 

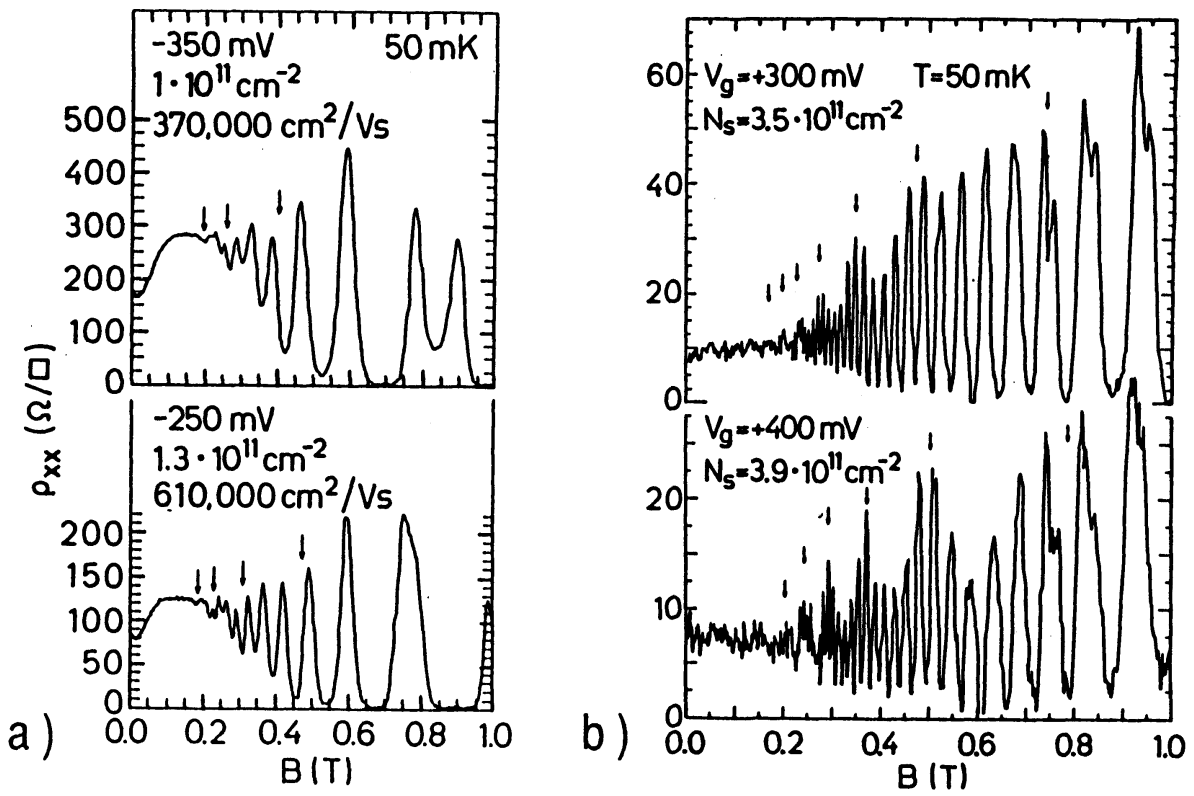

Fig. 8 Oscillations of the low ficld SdH-amplitudes at low (a) and high (b) mobility, measured at $50 \mathrm{mK}$. The change in mobility was achicved by biasing the micropatterned gate $(a=150 \mathrm{~nm})$ positively. The arrow positions mark nat bands (Eq. (1)) and are determined by the carricr density $n_{s}$ and period $a$. While $\rho_{x x}$ displays minima at the arrow position in (a), maxima are observed in (b) where the mobility is increased to $\sim 2 \times 10^{6} \mathrm{~cm}^{2} /$ Vs.

\section{Strong Modulation: Antidot Superlattices}

Antidot systems, geometrically complementary to quantum dot systems (sec, c.g., [27,28]), have attracted recent interest $[29,30,31]$. By ctching an array of holes through the two-dimensional electron gas the clectrons are forced to move between periodically arranged, disc shaped, strong repulsive potentials. The clectron wavefunction must vanish at antidot positions. Im the classical limit of frec particles, scattered between specularly reflecting dises, the system is known as Sinai's billiard (sec, c.g., [32]). The sheet resistance at $B=0, R_{\square}$, is detcrmined by scattcring from the antidots. For clectrons "channcling" through the superlattice, however, the electron mean frec path is still comparable to the one measured in the unpatterned 2DEG, and much longer than the period $a$. In contrast to weakly ID- and 2D-modulated systems the encrgy spectrum is not known in the presence of a magnetic field. Neither the Hofstadter picture, starting from a tight-binding, cosine electron dispersion, nor the weak periodic 
potential description are an appropriate approach. It turns out, however, that the origin of the low- $B$ features observed in experiment so far can be described in a picture involving commensurate orbits but not orbit quantization [33]. Data of Fig. 9 demonstrate that the imposed array of antidots dramatically affects transport at low- $B$. As expected, $R_{\square}$ increases after patterning and the low- $B$ mobility is drastically reduced. The corresponding electron mean frec path drops from $\ell \sim 7 \mu \mathrm{m}$ to a valuc $\ell^{\prime} \sim 0.34 \mu \mathrm{m}$ after patterning and has become comparable to $a$. Characteristic for the system are the pronounced peaks manifested at low magnetic ficlds (Fig. 9(a)), the accompanying (non-quantized) stcps in $\rho_{x y}$ (Fig. $9(\mathrm{~b})$ ), and the quenching of the Hall resistance about $B=0$ (Fig. 9(b), inset). Arrows in Fig. 9, which closely correlate with these features, mark ficld positions where the cyclotron radius, $R_{c}$, equals $a / 2$ and $3 a / 2$. When $R_{c}<a / 2, \rho_{x x}$ drops precipitously, quantum oscillations cmergc and $\rho_{x y}$ begins to display quantum Hall platcaus. In this regime traces from patterned and unpatterned segments become essentially identical. This suggests that the intrinsic mobility is preserved after patterning. Magnetoresistance curves from three samples are compared in Fig. 10. A progressively greater number of peaks in $\rho_{x x}$, and steps in $\rho_{x y}$, are resolved in traces with smaller $R_{\square}$. This is essentially

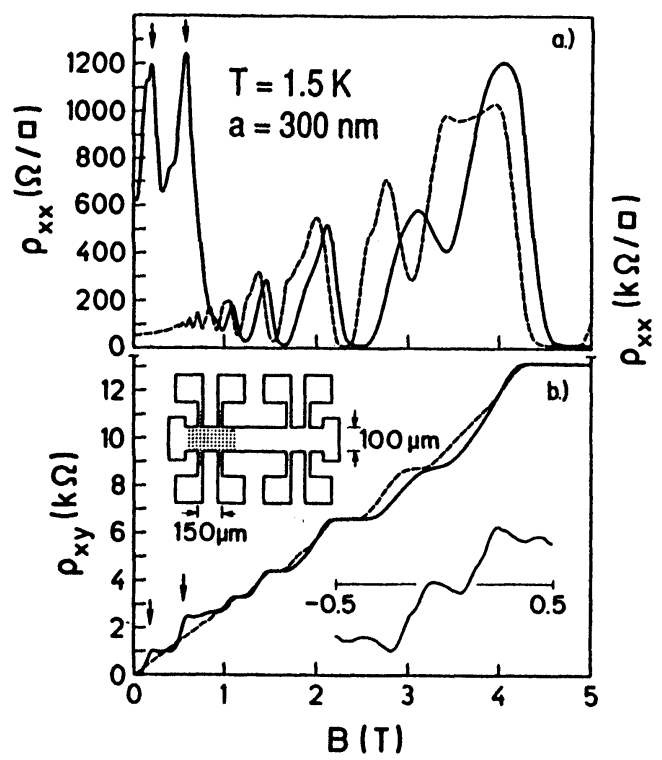

Fig. 9 Magnetoresistance (a) and Hall resistance (b) measured in patterned (solid line) and unpatterned (dashed line) sample segments of the sample geometry sketched in the top inset of (b). The bottom inset magnifics the quench in $\rho_{x y}$ about $B=0$ (from [33]). 


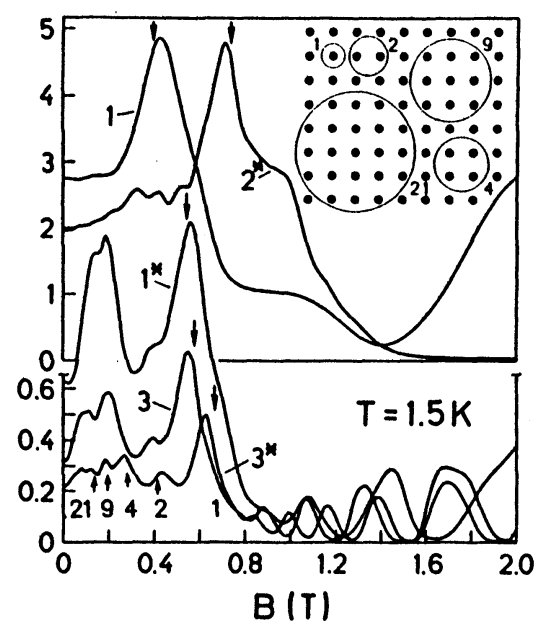

Fig. 10 Low- $B$ anomalics from samples of three different heterojunctions. Arrows mark $R_{c}=a / 2$ for cach tracc. Illumination of Sample $1\left(1 \rightarrow 1^{*}\right)$ increases $n_{s}$ by only a small factor $(\sim 1.7)$, whereas $R_{\square}$ drops almost by a factor of 5 . This indicates that the depletion length and, consequently, $\hat{d}$ are reduced after illumination. For smaller $\hat{d}$ more structure in $\rho_{x x}$ becomes resolved. Pcaks in tracc $3^{*}$ can be ascribed to commensurate orbits with $n=1,2,4,9,21$; as sketched in the inset (for $\hat{d}=1 / 3$ and average $R_{c} / a=0.5,0.8,1.14,1.7,2.53$; respectively) (from [33]).

controlled by the normalized antidot cross-section, $\hat{d}=d / a$, including the depletion length, and the carrier density $n_{s}$. Data from sample $3^{*}$, involving the lowest $R_{\square}$ and the smallest $\hat{d}$ (estimated to be $\sim 1 / 3$ ) displays the largest sequence of peaks in $\rho_{x x}$, and corresponding platcaus in $\rho_{x y}$. The structure is aperiodic in $1 / B$, but cach peak can be associated with a particular commensurate orbit for well defined magnetic field values. These special trajectories encircle a specific number of antidots (Fig. 10, inset) and in a simple model [33], clectrons on such orbits are assumed to be pinned. The repulsive potential at cach antidot provides a local restoring force against drift induced by external clectric ficlds. The "removal" of an electron from transport requires a long pinned orbit lifetime, $\tau_{\text {pin }}>\ell^{\prime} / v_{F}$, obtained when the initial mobility is preserved between antidots. This condition is fullfilled: the peak associated with an orbit around 9 antidots (Fig. 10 , trace $3^{*}$ ), c.g., requires an unscattered trajectory of $3.2 \mu \mathrm{m}$ (perimeter) and provides a second, independent proof that the electron gas between the antidots is nearly devoid of defects. The low ficld structure in $\rho_{x x}$ and $\rho_{x y}\left(2 R_{c}>a-d\right)$ can be understood in terms of Drude's model where in unpatterned material $\rho_{x x}=m^{*} / e^{2} \tau n_{s}\left(\equiv \rho_{0}\right)$, and $\rho_{x y}=B / e n_{s}\left(\equiv B R_{0}\right)$ holds. A fraction $f_{p}$ of clectrons 


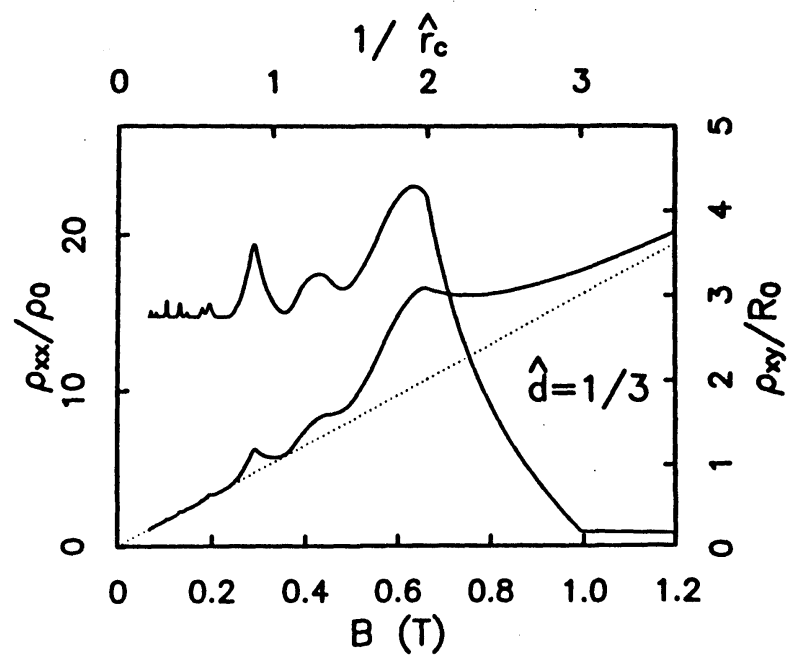

Fig. 11 Calculated magneto- and Hall resistance assuming a constant clectron mean free path $\ell^{\prime} / a \sim 2.4$, and an intrinsic value $\ell / a \sim 33$ (Values taken from experiment). Features attributed to orbits around 1,2 , and 4 antidots are most prominent. Top axis is in units of the normalized cyclotron radius $\hat{r}_{c} \equiv R_{c} / a$. The fraction of pinned orbits, for given $\hat{r}_{c}$, is obtained by calculating numcrically the fraction of completed clectron orbits with centers on a grid of $\sim 10^{5}$ sites within the real space unit cell (from [33]).

removed from transport increases both, $\rho_{x x}$ and $\rho_{x y}$ for commensurate magnetic ficlds. The current through the device, on the other hand, is carricd by electrons scattered as in pinball. This fraction $f_{s}$, scatters with an effective relaxation rate $\tau^{\prime}=\left(\tau^{-1}+\tau_{e x}^{-1}\right)^{-1}$ where the index "ex" denotes the scattering from the antidot lattice. At higher $B$ drifting orbits $\left(f_{d}\right)$ become important: when $2 R_{c}$ becomes smaller than $a-d$ backscattering is suppressed and transport is again characterized by the intrinsic scattering rate $\tau$. This is the reason for the strong negative magnetoresistance observed experimentally. With this ingredients expessions for $\rho_{x x}$ and $\rho_{x y}$, only dependent on the different fractions $\tau$ and $\tau^{\prime}$ [33], can be found. A model calculation using the parameters for sample $3^{*}$ in Fig. 10 nicely reproduces the experimental low ficld result. Based on simple geometrical considerations, the different fractions have been determined numerically [33]. For higher values of $R_{\square}$ deviations from the simple circular orbit picture are observed: the peak at $R_{c} \sim 3 a / 2$ (Fig. 9, Fig. 10), emerging from the circular orbits around 9 and 4 antidots, is forbidden for circular orbits. The nonvanishing slope of the potential between the antidots secms to leave its mark and arises interesting questions for future rescarch. 


\section{Concluding Remarks}

The interplay between magnetic length and period $a$ creates a varicty of interesting commensurability phenomena, both in the weak and strong modulation limit. Only a sketch of the existing theoretical and experimental work could be given herc. Many interesting issucs as magnetic breakdown [34,35], van Hove singularities [7] and the quenching of the Hall effect in lateral superlattices have been omitted. The reference list is by no means complete and a lot of interesting physics can be found in the reference lists of the cited literature.

\section{Acknowledgements}

It is a plcasure to thank K. von Klitzing, R. R. Gcrhardts, D. Pfannkuche, U. Wulf, T. Geisel, and R. Blümel for helpful discussions. I highly appreciate the cooperation with A. Menschig on electron beam patterning and the help of $\mathrm{P}$. Grambow in etching the antidot samples. I wish to thank M. Rick and the Servicegruppe Technologic at the Max-Planck Institut for assistance in sample preparation, S. Koch for supporting the millikelvin measurements, and $\mathrm{K}$. Ploog, G. Wcimann, and $\mathrm{H}$. Nickel for providing $\mathrm{mc}$ with high quality samples. I further acknowledge very fruitful cooperation with M. L. Roukes to model the antidot system.

\section{References}

[1] D. R. Hofstadter, Phys. Rev. B 14, 2239 (1976); and references thercin

[2] P. Drude, Annalen der Physik 1, 566 and 3, 369 (1900)

[3] T. Ando and Y. Uemura, J. Phys. Soc. Jpn. 36, 959 (1974)

[4] R. R. Gerhardts, Z. Phys. B 21, 285 (1975)

[5] K. von Klitzing, G. Dorda, and M. Pepper, Phys. Rev. Lett. 45, 494 (1980)

[6] D. Weiss, K. von Klitzing, K. Ploog, and G. Weimann, Europhys. Lett. 8, 179 (1989); also in: High Magnetic Ficlds in Semiconductor Physics II, Vol. 87 of Springer Series in Solid State Sciences, ed. by G. Landweltr (Springer, Bcrlin 1989), p. 357

[7] D. Weiss, K. von Klitzing, and K. Ploog, Surf. Sci. 229, 88, (1990)

[8] D. Weiss, P. Grambow, K. von Klitzing, A. Menschig, and G. Weimann, Appl. Phys. Lett., (24. Junc 91)

[9] R. W. Winkler, J. P. Kotthaus, and K. Ploog, Phys. Rev. Lett. 62, 1177 (1989)

[10] E. S. Alves, P. H. Beton, M. Henini, L. Eaves, P. C. Main, O. H. Hughes, G. A. Toombs, S. P. Beaumont, and C. D. W. Wilkinson, J.Phys. Condens. Matter 1, 8257 (1989)

[11] H. Fang and P..J. Stiles, Phys. Rev. B 41, 10171 (1990)

[12] P. H. Beton, E. S. Alves, P. C. Main, L. Eaves, M. W. Dellow, M. Henini, $O$. H. Hughes, S. P. Beaumont, and C. D. W. Wilkinson, Phys. Rev. B 42, 9229 (1990) 
[13] R. R. Gerhardts, D. Weiss, and K. von Klitzing, Phys. Rev. Lett. 62, 1173 (1989)

[14] P. H. Beton, P. C. Main, M. Davison, M. Dellow, R. P. Taylor, E. S. Alves, L. Eaves, S. P. Beaumont and C. D. W. Wilkinson, Phys. Rev. B 42, 9689 (1990)

[15] C. Zhang and R. R. Gerhardts, Phys. Rev. B 41, 12850 (1990)

[16] V. Mosser, D. Weiss, K. von Klitzing, K. Ploog, and G. Weimann, Solid Statc Commun. 58, 5 (1986); D. Weiss and $K$. von Klitzing, High Magnetic Ficlds in Semiconductor Physics, Vol. 71 of Springer Serics in Solid State Sciences, ed. by G. Landwehr (Springer, Berlin 1987), p. 57

[17] D. Weiss, C. Zhang, R. R. Gerhardts, and K. von Klitzing, Phys. Rev. B 39, 13020 (1989)

[18] K. Ismail, T. P. Smith, III, W. T. Masselink, and H. I. Smith, Appl. Phys. Lett. 55, 2766 (1989)

[19] P. Vasilopoulos and F. M. Peeters, Phys. Rev. Lett. 63, 2120 (1989)

[20] F. M. Peeters and P. Vasilopoulos, Phys. Rcv. B 42, 5899 (1990)

[21] H. L. Cui, V. Fessatidis, and N. J. M. Horing, Phys. Rev. Lett. 63, 2598 (1989)

[22] F. Brinkop, C. Dahl, J. P. Kotthaus, G. Weimann, and W. Schlapp, High Magnetic Ficlds in Semiconductor Physics III, Springer Scrics in Solid State Sciences, ed. by $G$. Landwehr (Springer, Berlin 1991), in press

[23] C. W. J. Beenakker, Phys. Rev. Lett. 62, 2020 (1989)

[24] R. R. Gerhardts, D. Weiss, and U. Wulf, Phys. Rev. B 43, 5192 (1991)

[25] A. Rauh, Phys. Status Solidi B 65, K9 (1975)

[26] R. R. Gerhardts, D. Pfannkuche, D. Weiss, and U. Wulf, High Magnetic Ficlds in Scmiconductor Physics III, Springer Scries in Solid State Sciences, cd. by G. Landwehr (Springer, Berlin 1991), in press

[27] Ch. Sikorsky and U. Merkt, Phys. Rev. Lett. 62, 2164 (1989)

[28] T. Demel, D. Heitmann, P. Grambow, and K. Ploog, Phys. Rev. Lett. 64, 788 (1990)

[29] K. Ensslin and P. M. Petroff, Phys. Rev. B 41, 12307 (1989)

[30] A. Lorke, J. P. Kotthaus, and K. Ploog, Superlattices and Microstructures 9, $103(1991)$

[31] K. Kern, D. Heitmann, P. Grambow, Y. H. Zhang, and K. Ploog, Phys. Rev. Lett. 66, 1618 (1991)

[32] M. V. Berry, Annals of Physics 131, 163 (1981); and references therein

[33] D. Weiss, M. L. Roukes, A. Menschig, P. Grambow, K. von Klitzing, and G. Weimann, to be published

[34] P. Streda and A. H. MacDonald, Phys. Rev. B 41, 11892 (1990)

[35] D. Weiss, Physica Scripta, in press (1991) 